\title{
Clinical value of TV-CDS combined with serum tumor markers in diagnosis of ovarian cancer
}

\author{
CHUNYING YU, TING DOU, YUN LIU and RUIRONG LIU \\ Department of Ultrasonic Medicine, The Third People's Hospital of Qingdao, \\ Qingdao, Shandong 266041, P.R. China
}

Received December 9, 2019; Accepted April 14, 2020

DOI: 10.3892/ol.2020.11705

\begin{abstract}
Clinical application value was investigated of transvaginal color Doppler ultrasound (TV-CDS) combined with serum tumor markers carbohydrate antigen 125 (CA125), vascular endothelial growth factor (VEGF) and osteopontin $(\mathrm{OPN})$ in the diagnosis of ovarian cancer (OC). One hundred and six patients with OC [malignant tumor group (MTG)] and fifty patients with benign ovarian diseases [benign control group (BCG)] were selected. Both groups of patients underwent TV-CDS examination. The lesion morphology and internal structure were observed, and the tumor blood flow signal, resistance index (RI) and pulsability index (PI) under ultrasound were determined. Serum CA125 was detected by electrochemiluminescence, and VEGF and OPN levels were detected by enzyme-linked immunosorbent assay. The incidence of irregular lesion morphology, unclear boundary, uneven internal echo, microcalcification and side-acoustic images in OC group (OCG) was significantly higher than that in BCG $(\mathrm{P}<0.01)$. As for blood flow grading, most patients in the MTG were in grade II and III, while most patients in the BCG were in grade 0 . Compared with $\mathrm{BCG}$, the flow RI and PI in the OCG were significantly reduced $(\mathrm{P}<0.01)$. The levels of serum CA125, VEGF and OPN in OCG were significantly higher than those in BCG. The expression levels of serum CA125, VEGF and OPN in OC patients with clinical high stage (stage III and IV), poorly differentiated, ascites, recurrence and metastasis were significantly higher than those in patients with clinical low stage (stage I and II), well differentiated, no ascites and no recurrence and metastasis $(\mathrm{P}<0.05)$. With the disappearance of the tumor or the decrease of tumor load, the serum marker levels after treatment were significantly lower than that before treatment $(\mathrm{P}<0.05)$. The sensitivity and accuracy of the combined examination in the diagnosis of $\mathrm{OC}$ were obviously improved compared with the single and partial combined examinations
\end{abstract}

Correspondence to: Dr Ruirong Liu, Department of Ultrasonic Medicine, The Third People's Hospital of Qingdao, 29 Yongping Road, Qingdao, Shandong 266041, P.R. China

E-mail: rsm466@163.com

Key words: ovarian cancer, color doppler ultrasound, transvaginal, tumor markers, serology, combined examination
$(\mathrm{P}<0.05)$. In conclusion, combined examination can significantly improve the sensitivity and accuracy of OC, which is conducive to early diagnosis and clinical intervention of OC.

\section{Introduction}

Ovarian cancer (OC) is one of the common malignant tumors in the female reproductive system. Its incidence rate ranks third below cervical cancer and carcinoma of corpus uteri, and its mortality rate ranks first among all kinds of gynecologic tumors, posing a serious threat to women's health (1). As the ovary is located in the deep part of the pelvic cavity, early symptoms are not typical. Most patients have developed middle and late stage OC when they seek medical treatment, missing the best treatment opportunity (2).

At present, the conventional methods for clinical screening of OC are trans-abdominal sonography and the determination of serum tumor marker carbohydrate antigen 125 (CA125). However, trans-abdominal sonography has many influencing factors, and the ratio of false positive and false negative in diagnosis of $\mathrm{OC}$ are high, resulting in poor stability and accuracy. The detection of serum CA125 alone has poor sensitivity and accuracy, so the early diagnosis of OC is not ideal (3). In recent years, transvaginal color doppler ultrasound (TV-CDS) technology is an emerging examination method, which can obtain clearer mass morphology, internal echo and blood flow images, providing reference for the diagnosis of OC by measuring blood flow parameters (4). At the same time, with the rapid development of molecular biology, an increasing number of tumor markers are closely related to $\mathrm{OC}$, such as CA125, vascular endothelial growth factor (VEGF), and osteopontin (OPN).

In this study, TV-CDS combined with serum CA125, VEGF and OPN were selected to diagnose OC, so as to provide basis for early diagnosis, monitor of disease progression and evaluation of prognosis of OC.

\section{Patients and methods}

Clinical materials. From July 2017 to December 2018, 106 patients with ovarian cancer (MTG) and 50 patients with benign ovarian diseases (BCG) who received initial treatment in The Third People's Hospital of Qingdao (Qingdao, China) were selected as the study subjects. Patients in MTG were aged 35-83 years, with an average age of 56.72 \pm 9.34 . 
Table I. Comparison of conventional ultrasonographic features of lesion location of patients in the two groups [n (\%)].

\begin{tabular}{lcccccc}
\hline Group & Cases & $\begin{array}{c}\text { Irregular } \\
\text { morphology }\end{array}$ & $\begin{array}{c}\text { Unclear } \\
\text { boundary }\end{array}$ & Uneven echo & $\begin{array}{c}\text { Micro- } \\
\text { calcification }\end{array}$ & $\begin{array}{c}\text { Side-acoustic } \\
\text { images }\end{array}$ \\
\hline MTG & 106 & $100(94.34)^{\mathrm{a}}$ & $77(72.64)^{\mathrm{a}}$ & $76(71.70)^{\mathrm{a}}$ & $73(68.87)^{\mathrm{a}}$ & $70(66.04)^{\mathrm{a}}$ \\
BCG & 50 & $3(6.00)$ & $2(4.00)$ & $3(6.00)$ & $1(2.00)$ & $2(4.00)$ \\
$\chi^{2}$ & & 118.195 & 64.041 & 58.666 & 60.924 & 52.614 \\
P-value & $<0.001$ & $<0.001$ & $<0.001$ & $<0.001$ & $<0.001$ \\
\hline
\end{tabular}

${ }^{\mathrm{a}} \mathrm{P}<0.01$, compared with BCG. MTG, malignant tumor group; BCG, benign control group.

Clinical staging referred to the Federation International of Gynecology and Obstetrics (FIGO) staging standard in 2009: A total of 11 cases in stage I, 39 cases in stage II, 44 cases in stage III and 12 cases in stage IV. The histopathological types were 69 cases of ovarian serous adenocarcinoma, 19 cases of ovarian mucinous adenocarcinoma, 11 cases of endometrial carcinoma and 7 cases of clear cell carcinoma. According to WHO classification criteria, there were 23 cases of well differentiated, 35 cases of middle differentiation and 48 cases of undifferentiated. Patients in BCG were 34-86 years of age, with an average age of $57.26 \pm 10.98$, including 29 cases of serous cystadenoma, 10 cases of mucinous cystadenoma, 6 cases of ovarian teratoma and 5 cases of endometrial cyst.

Inclusion criteria were: i) Patients treated for the first time without radiotherapy and chemotherapy or endocrine therapy; ii) patients who underwent TV-CDS, serum CA125, VEGF and OPN; iii) patients who underwent surgery or laparoscopic treatment in the hospital and were diagnosed by pathology and iv) patients with complete follow-up data.

Exclusion criteria were: i) Incomplete medical history data; ii) other important organ dysfunction and iii) combined with other tumors.

The study was approved by the Ethics Committee of The Third People's Hospital of Qingdao. All patients who participated in this research had complete clinical data. Patients provided a signed informed consent and volunteered to participate in the study. Age and other baselines of the two groups were not statistically significant, and thus, the groups were comparable.

TV-CDS examination. PHILIPS iU22 (Koninklijke Philips N.V.) was used for color doppler ultrasound diagnosis, with a transducer frequency of 5-7 MHz. The patient was instructed to empty the bladder before examination. During examination, the patient lay on the back in a lithotomy position. The transvaginal ultrasound probe was covered with a disposable latex cot or condom. The cot was coated with disinfectant couplant. The probe was placed in the deepest part of the vagina. The size, morphology, boundary, internal echo, calcification, side-acoustic images and other imaging conditions of the lesion were explored (5). Then the blood flow were observed using color doppler flow imaging (CDFI) technology, including flow classification, resistance index (RI) and pulsability index (PI). Flow classification (6): Grade 0: there was no blood flow signal; Grade I: There was punctiform or short line blood flow around the tumor but there was no blood flow signal inside the tumor. Class II: There was punctiform or short line blood flow inside the tumor, with regular blood vessel course; Class III: the blood flow inside the tumor was dendritic and reticular, with abundant short and long blood flow, tortuosity and disordered blood vessels. RI and PI were directly read out and counted by ultrasonic instruments. The ultrasonography, recording and image diagnosis of all patients were determined by two deputy directors of the ultrasonography department.

Examination of serum tumor markers. A total of $4 \mathrm{ml}$ fasting elbow venous blood was extracted from OC patients before treatment and at 3 months after treatment, and from the subjects in the BCG group at 6.00-9.00 in the morning. The serum was self-coagulated at room temperature, and then centrifuged using DT5-4 automatic decap centrifuge (Beijing Era Beili Centrifuge Co., Ltd.) at 2,264 x g, at room temperature for $30 \mathrm{~min}$. CA125 was detected by electrochemiluminescence using Roche ELecsys-2010 and Roche original reagents. VEGF and OPN were detected by enzyme-linked immunosorbent assay (ELISA). Reagents were provided by R\&D Systems, Inc. Tecan Infinite M1000 PRO multi-function enzyme-labeling instrument (Tecan Group, Ltd.) was used according to the manufacturer's instructions. The reference values were CAl25 $\leq 35.00 \mathrm{U} / \mathrm{ml}$, VEGF $\leq 148.60 \mathrm{pg} / \mathrm{ml}$ and $\mathrm{OPN} \leq 49.90 \mathrm{ng} / \mathrm{ml}$.

Result estimation. One or more positives in the combined examination were considered positive. All negatives were considered as negative.

Evaluation index. The standard method of diagnostic test evaluation: Subjects were divided into patients and nonpatients by gold standard diagnosis, a certain test method was used to measure the positive and negative results, and then various statistical analysis were carried out. Based on this, the evaluation indexes commonly used in diagnostic tests can be calculated. The results of the test diagnosis were divided into (a) true positive, (b) false positive, (c) false negative and (d) true negative. Calculation formula: Sensitivity $=a /(a+c)$; Specificity $=\mathrm{d} /(\mathrm{d}+\mathrm{b})$; Accuracy $=(\mathrm{a}+\mathrm{d}) /(\mathrm{a}+\mathrm{b}+\mathrm{c}+\mathrm{d})$; Positive predictive value $=a /(a+b) ;$ Negative predictive value $=d /(d+c)$.

Statistical analysis. Excel 2007 was used to establish a database. SPSS17.0 statistical software, t-test and $\chi^{2}$ test were applied. The concentration level of the measured data was expressed as mean \pm standard deviation (SD). The comparison of the mean number between groups was conducted by independent sample $\mathrm{t}$-test. The comparison of the counting data rate was conducted by $\chi^{2}$ test, with statistical significance $(\mathrm{P}<0.05)$. 
A

\section{B}
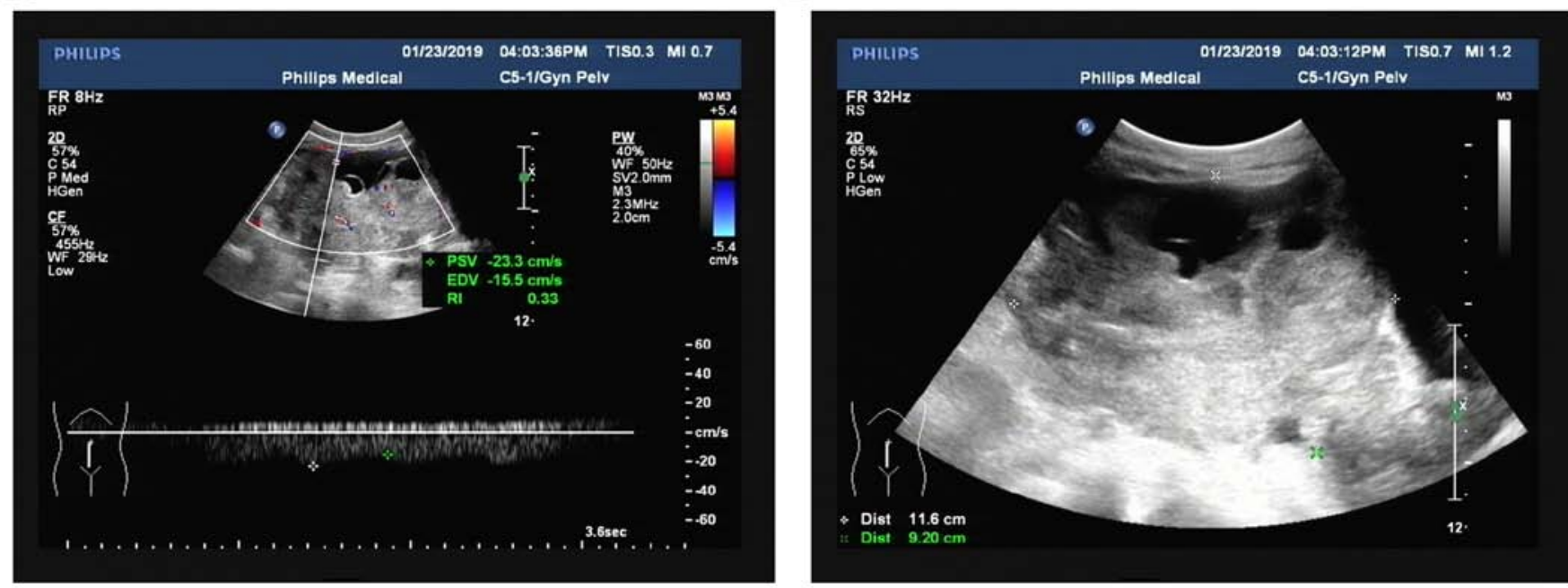

Figure 1. Ultrasonic acoustic and blood flow display images of ovarian cancer. (A) Ultrasonic acoustic image of ovarian cancer. (B) Blood flow display image of ovarian cancer. Ovarian cancer: the size of the tumor was $11.6 \times 9.2 \times 10.2 \mathrm{~cm}$, the shape was irregular, the boundary was unclear, the boundary was irregular and solid was the main feature. CDFI of its internal detection and rich blood flow signal and RI was 0.33. CDFI, color doppler flow imaging; RI, resistance index.

Table II. Comparison of serum CDFI of patients between OCG and the control group.

\begin{tabular}{|c|c|c|c|c|c|c|c|}
\hline \multirow[b]{2}{*}{ Group } & \multirow[b]{2}{*}{ Cases } & \multirow{2}{*}{$\begin{array}{c}\text { RI } \\
(\text { mean } \pm \mathrm{SD})\end{array}$} & \multirow{2}{*}{$\begin{array}{c}\text { PI } \\
(\text { mean } \pm \text { SD })\end{array}$} & \multicolumn{4}{|c|}{ Blood flow grading [n (\%)] } \\
\hline & & & & 0 & I & II & III \\
\hline MTG & 106 & $0.38 \pm 0.04^{\mathrm{a}}$ & $0.63 \pm 0.09^{\mathrm{a}}$ & $0(0)^{\mathrm{a}}$ & $10(9.43)^{\mathrm{a}}$ & $61(57.55)^{\mathrm{a}}$ & $35(33.02)^{\mathrm{a}}$ \\
\hline BCG & 50 & $0.73 \pm 0.09$ & $1.25 \pm 0.15$ & $35(70.00)$ & $12(24.00)$ & $3(6.00)$ & $0(0.00)$ \\
\hline
\end{tabular}

${ }^{a} \mathrm{P}<0.01$, compared with BCG. CDFI, color Doppler flow imaging; OCG, ovarian cancer group; RI, resistance index; PI, pulsability index; MTG, malignant tumor group; BCG, benign control group.

Table III. Comparison of serum CA125, VEGF and OPN levels of patients between OCG and the control group (mean \pm SD).

\begin{tabular}{lrccccccccc}
\hline Group & Cases & CA125 $(\mathrm{U} / \mathrm{ml})$ & t-test & P-value & VEGF $(\mathrm{pg} / \mathrm{ml})$ & t-test & P-value & OPN $(\mathrm{ng} / \mathrm{ml})$ & t-test & P-value \\
\hline MTG & 106 & $169.82 \pm 26.38^{\mathrm{a}}$ & 5.981 & 0.001 & $679.62 \pm 57.83^{\mathrm{a}}$ & 10.288 & $<0.001$ & $216.87 \pm 34.95^{\mathrm{a}}$ & 5.645 & 0.001 \\
BCG & 50 & $22.83 \pm 7.29$ & & & $123.54 \pm 16.68$ & & & $36.62 \pm 8.73$ & &
\end{tabular}

${ }^{a} \mathrm{P}<0.01$, compared with BCG. CA125, carbohydrate antigen 125; VEGF, vascular endothelial growth factor; OPN, osteopontin; OCG, ovarian cancer group; MTG, malignant tumor group; BCG, benign control group.

\section{Results}

Sonographic comparison of $O C$ and benign ovarian diseases by TV-CDS. According to the histopathological 'gold standard' and the diagnostic value of TV-CDS for benign and malignant ovarian tumors, the sensitivity of TV-CDS in the diagnosis of OC was $75.47 \%$ (80/106) and the specificity was $90.00 \%(45 / 50)$. TV-CDS showed that the incidence of lesions in conventional two-dimensional ultrasound including irregular morphology, unclear boundary, uneven echo, microcalcification and sideacoustic images in OCG was significantly higher than that in BCG $(\mathrm{P}<0.01)$, as shown in Table I. Regarding blood flow grading, most patients in the MTG were in grade II and III, while most patients in the BCG were in grade 0. Compared with RI $(0.73 \pm 0.09)$ and PI $(1.25 \pm 0.15)$ in BCG, RI $(0.38 \pm 0.04)$ and PI $(0.63 \pm 0.09)$ in the OCG were significantly decreased $(\mathrm{t}=-3.893,-3.883, \mathrm{P}<0.01)$ (Table II). The ultrasonic acoustic and blood flow display images of OC are shown in Fig. 1.

Comparison of serum CA125, VEGF, OPN levels of patients between OCG and the control group. The levels of serum CA125, VEGF and OPN of patients in OCG were significantly higher than those in BCG, with statistically significant difference $(\mathrm{P}<0.01)$ (Table III). 


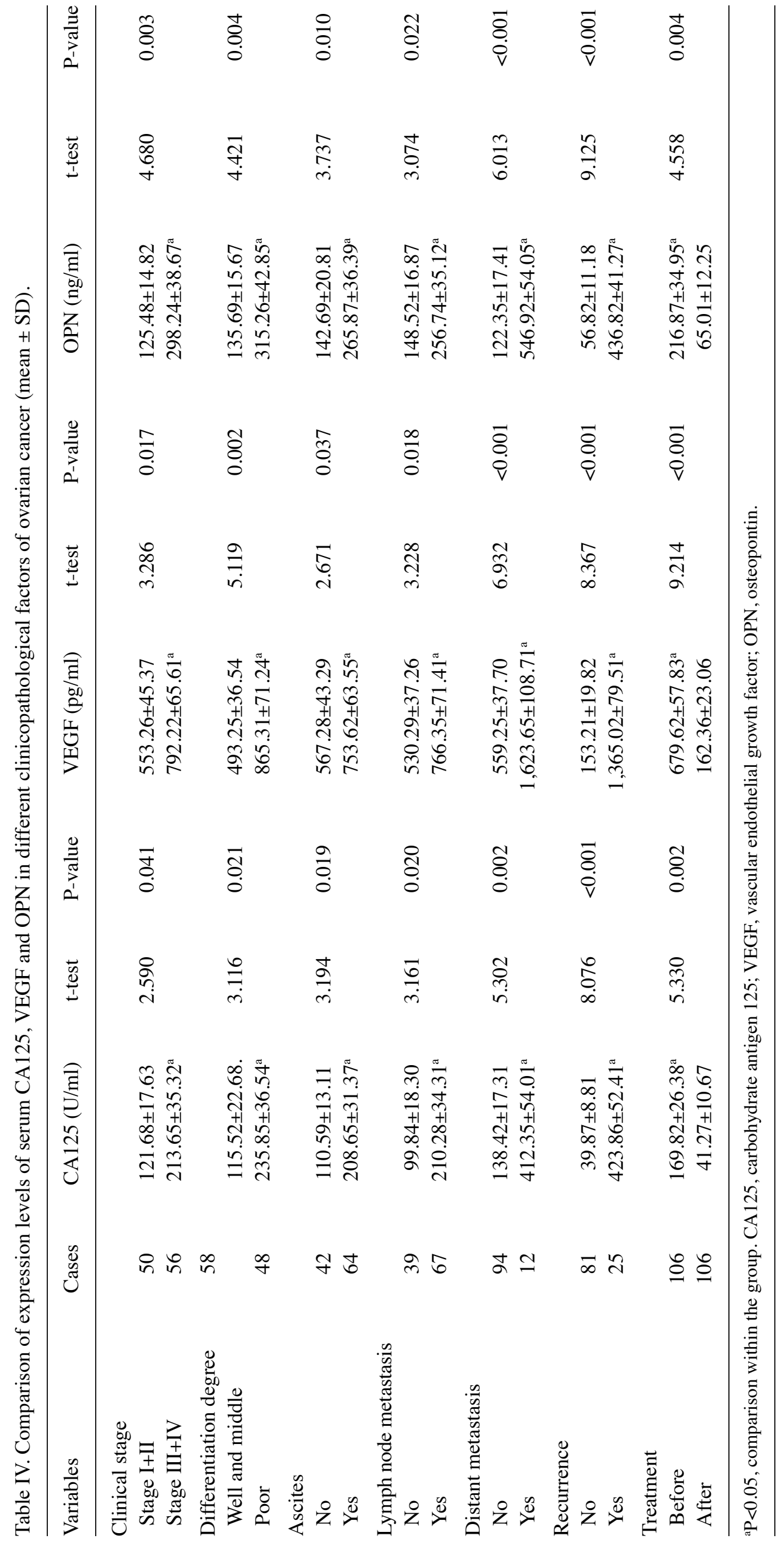


Table V. Diagnostic value of transvaginal ultrasound, CA125, VEGF, OPN single and combined examination in ovarian cancer [\% (ratio)].

\begin{tabular}{lccccc}
\hline Detection indexes & Sensitivity & Specificity & Accuracy & $\begin{array}{c}\text { Positive } \\
\text { predictive value }\end{array}$ & $\begin{array}{c}\text { Negative } \\
\text { predictive value }\end{array}$ \\
\hline Ultrasound & $75.47(80 / 106)$ & $90.00(45 / 50)$ & $80.12(125 / 156)$ & $94.12(80 / 85)$ & $63.38(45 / 71)$ \\
CA125 & $60.38(64 / 106)$ & $94.00(47 / 50)$ & $71.15(111 / 156)$ & $95.52(64 / 67)$ & $52.81(47 / 89)$ \\
VEGF & $58.49(62 / 106)$ & $96.00(48 / 50)$ & $70.51(110 / 156)$ & $96.88(62 / 64)$ & $52.17(48 / 92)$ \\
OPN & $55.66(59 / 106)$ & $92.00(46 / 50)$ & $67.31(105 / 156)$ & $93.65(59 / 63)$ & $49.46(46 / 93)$ \\
CA125+VEGF+OPN & $80.19(85 / 106)$ & $88.00(44 / 50)$ & $82.69(129 / 156)$ & $93.41(85 / 91)$ & $67.69(44 / 65)$ \\
Ultrasound+CA125 & $97.17(103 / 106)^{\mathrm{a}}$ & $84.00(43 / 50)$ & $93.59(146 / 156)^{\mathrm{a}}$ & $96.26(103 / 107)$ & $93.48(43 / 46)^{\mathrm{a}}$ \\
+VEGF+OPN & & & & & \\
\hline
\end{tabular}

${ }^{a} \mathrm{P}<0.05$, compared with individual and partial combination tests. CA125, carbohydrate antigen 125; VEGF, vascular endothelial growth factor; OPN, osteopontin.

Comparison of expression levels of serum CA125, VEGF and $O P N$ in different clinicopathological factors of $O C$. The expression levels of serum CA125, VEGF and OPN in OC patients with clinical high stage (stage III and IV), poorly differentiated, ascites, recurrence and metastasis were significantly higher than those in patients with clinical low stage (stage I and II), well differentiated, no ascites and no recurrence and metastasis $(\mathrm{P}<0.05)$. With the disappearance of the tumor or the decrease of tumor load, the serum marker levels after treatment were significantly lower than those before treatment $(\mathrm{P}<0.05)$ (Table IV).

Comparison of diagnostic value of transvaginal ultrasound, CA125, VEGF, OPN single and combined examination in $O C$. The sensitivity, accuracy and negative predictive value of the combination of transvaginal ultrasound, CA125, VEGF and OPN in the diagnosis of OC were significantly improved compared with those of individual and partial combination tests. The differences were statistically significant $(\mathrm{P}<0.05)($ Table V).

\section{Discussion}

Compared with the traditional transvaginal color Doppler imaging (TV-CDS), the transvaginal ultrasound is closer to the ovary. The frequency of the probe is higher without interference from abdominal wall fat and flatulence, which significantly improves image quality and resolution and makes it more objective and accurate to judge whether the ovarian tumors are benign or malignant. Schelling et al (4) stated that two important factors for the diagnosis of $\mathrm{OC}$ by ultrasound are the determination of the solid component of the lesion and the presence of blood flow signals in the solid component. The benign and malignant ovarian masses were judged by color Doppler ultrasound two-dimensional image and blood flow imaging (7). The results of this study showed that the incidence of irregular lesion shape, unclear boundary, uneven internal echo, microcalcification and lateral sound shadow in the OC group was significantly higher than that in the benign lesion group. Due to the lack of muscle layer in the neovasal wall of ovarian malignant tumor, the neovasal wall is highly permeable and the vascular wall is thin, presenting a low-resistance spectrum, and the RI and PI of OC are significantly lower than those of benign ovarian tumors (8). Kurjak et al (9) proposed that the sensitivity of $\mathrm{RI} \leq 0.4$ in the diagnosis of ovarian malignancy was $86.4 \%$. The results of this study showed that the sensitivity of TV-CD in the diagnosis of OC was $75.47 \%$, slightly lower than those in previous reports, which may be related to the low threshold of RI ( $\leq 0.35)$. A study on early detection of OC screening was carried out in the United States, 4,526 women at high risk of developing $\mathrm{OC}$ were screened by transvaginal ultrasound. The value of single detection method of screening was limited, in particular, early ovarian patients with low blood flow was easily misdiagnosed (10). Therefore, combined detection with other markers is needed.

The detection of serum tumor markers is a common detection method in the diagnosis of malignant tumors, because it is simple and inexpensive, and the samples are easy to collect. CA125 is still the most commonly used biomarker for the diagnosis and efficacy monitoring of $\mathrm{OC}$, providing valuable information for the determination of the benign and malignant ovarian tumors (11). It has been reported that CA125 is elevated in the serum of most ovarian epithelial carcinoma, and continuous elevation of CA125 level indicates a relatively poor prognosis (12). It was better in the prognosis of patients where CA125 level declined to normal after 3 months of treatment, compared with those who did not return to normal. The sensitivity of CA125 in the diagnosis of OC in this study was $60.38 \%$, which was relatively lower than the $73.60 \%$ reported by Schummer et al (13), which may be related to the difference in selected cases. The expression level of CA125 in mucous ovarian epithelial carcinoma is low, which is prone to false negatives. In female inflammatory diseases, ovarian chocolate cyst, follicular cyst, pregnancy and menstruation, CA125 is increased to different degrees (14), which affects the accurate diagnosis of $\mathrm{OC}$ and results in false positive, indicating that the value of CA125 alone is limited. VEGF is a cytokine that regulates angiogenesis and acts specifically on vascular endothelial cells. The role of VEGF in tumors is not limited to the formation of blood vessels and the regulation of vascular permeability (15). Previous research has shown that the 
presence of VEGF regulatory signal is found in tumor cells, which not only acts on the formation of tumor cells, but also plays a certain role in the formation of tumor stem cells and tumor metastasis (16). VEGF plays an important role in the formation of lymphatic vessels (17). As an important factor of tumor angiogenesis, VEGF plays a very important role in the occurrence, progression and invasion of tumors (16). Serum expression level is significantly correlated with the clinical stage, invasion, recurrence and metastasis of tumors (18). The results of this study showed that the sensitivity of VEGF in the diagnosis of OC was $58.49 \%$. Li et al (19) reported that the sensitivity of VEGF in the diagnosis of OC was $77.00 \%$ in the combined detection of VEGF and CA125, indicating that the value of VEGF alone was limited.

OPN is a glycosylated phosphorylated protein that is associated with the development, invasion and metastasis of tumors. Its expression is significantly up-regulated in a variety of malignant tumor tissues (20). In terms of the mechanism of tumor angiogenesis, VEGF can play a synergistic role with OPN to promote the generation of granulation tissue and induce angiogenesis. The establishment of new blood vessels can promote cell migration, adhesion and prevent cell apoptosis, which is conducive to tumor cell generation (21). The increase of OPN level in peripheral blood of advanced OC is more obvious, and the serum expression level is positively correlated with clinical stage (22). As for the mechanism of tumor invasion, metastasis and relapse, cell adhesion - GRGDS sequence in OPN may interact with integrin and cancer cell surface adhesion molecule CD44 to inhibit endothelial cell apoptosis, and cause a variety of protein dissolving enzyme synthesis and secretion, so as to dissolve the extracellular matrix barrier to tumorigenesis of invasion and metastasis (23). Relevant research has shown that OPN is closely correlated with the development of OC (21). Kim et al (24) studied 107 plasma samples by cDNA array and found that there was a significantly high level of OPN expression in invasive OC and ovarian junction tumors. It has been pointed out that OPN positive expression could be detected in OC patients with negative CA125, and the detection of OPN was complementary (25). The results of this study showed that the sensitivity of OPN in the diagnosis of OC was $55.66 \%$, indicating that the value of detection alone was limited. Combined detection with other markers can enhance the value.

The results of this study showed that transvaginal ultrasound and serum CA125, TSGF and VEGF have their respective advantages and disadvantages in the diagnosis of $\mathrm{OC}$, and the sensitivity and accuracy are not high, which is far from the clinical requirements. Because the combined parallel method was used, that is, if any of the indicators is positive, it is determined to be positive. Although the specificity decreased $(84.00 \%)$, the sensitivity, accuracy and negative predictive value $(97.17,93.59$, and $93.48 \%$, respectively) were significantly higher than those of individual examinations, thereby missed diagnosis was reduced.

In conclusion, TV-CDS combined with serum CA125, TSGF, and VEGF can be used as an effective method for the diagnosis of OC. The selected sample size for this study was limited. Therefore, research on larger sample size and prognostic follow-up will be necessary.

\section{Acknowledgements}

Not applicable.

\section{Funding}

No funding was received.

\section{Availability of data and materials}

The datasets used and/or analyzed during the current study are available from the corresponding author on reasonable request.

\section{Authors' contributions}

CY was involved in the conception of the study and wrote the manuscript. TD and YL collected and analyzed the general data of the patients. RL assisted with the statistical analysis. All authors read and approved the final manuscript.

\section{Ethics approval and consent to participate}

The study was approved by the Ethics Committee of The Third People's Hospital of Qingdao (Qingdao, China). All patients who participated in this research had complete clinical data. Signed informed consents were obtained from the patients and/or guardians.

\section{Patient consent for publication}

Not applicable.

\section{Competing interests}

The authors declare that they have no competing interests.

\section{References}

1. Torre LA, Bray F, Siegel RL, Ferlay J, Lortet-Tieulent J and Jemal A: Global cancer statistics, 2012. CA Cancer J Clin 65: 87-108, 2015.

2. Ulusoy S, Akbayir O, Numanoglu C, Ulusoy N, Odabas E and Gulkilik A: The risk of malignancy index in discrimination of adnexal masses. Int J Gynaecol Obstet 96: 186-191, 2007.

3. Menon U, Talaat A, Rosenthal AN, Macdonald ND, Jeyerajah AR, Skates SJ, Sibley K, Oram DH and Jacobs IJ: Performance of ultrasound as a second line test to serum CA125 in ovarian cancer screening. BJOG 121: 35-39, 2014.

4. Schelling M, Braun M, Kuhn W, Bogner G, Gruber R, Gnirs J, Schneider KT, Ulm K, Rutke S and Staudach A: Combined transvaginal B-mode and color Doppler sonography for differential diagnosis of ovarian tumors: Results of a multivariate logistic regression analysis. Gynecol Oncol 77: 78-86, 2000.

5. Goldberger S, Tepper R, Markov S and Beyth Y: Transvaginal sonographic characterization of ovarian disease: Evaluation of a new scoring system to predict ovarian malignancy. Obstet Gynecol 78: 1151-1152, 1991.

6. Kupesic S and Kurjak A: Contrast-enhanced, three-dimensional power Doppler sonography for differentiation of adnexal masses. Obstet Gynecol 96: 452-458, 2000.

7. Benjapibal M, Sunsaneevitayakul P, Boriboonhirunsarn D, Sutanthavibul A and Chakorngowit M: Color Doppler ultrasonography for prediction of malignant ovarian tumors. J Med Assoc Thai 85: 709-715, 2002.

8. Sawicki W, Spiewankiewicz B, Cendrowski K and Stelmachów J: Preoperative discrimination between malignant and benign adnexal masses with transvaginal ultrasonography and colour blood flow imaging. Eur J Gynaecol Oncol 22: 137-142, 2001. 
9. kurjak A, Kupesic S, Sparac V,Prka M and Bekavac I: The detection of stage I ovarian cancer by three-dimensional sonography and power Doppler. Gynecol Oncol 90: 258-264, 2003.

10. Fishman DA, Cohen L, Blank SV, Shulman L, Singh D, Bozorgi K, Tamura R, Timor-Tritsch I and Schwartz PE: The role of ultrasound evaluation in the detection of early-stage epithelial ovarian cancer. Am J Obstet Gynecol 192: 1214-1221, discussion $1221-1222,2005$

11. Gupta KK, Gupta VK and Naumann RW: Ovarian cancer: Screening and future directions. Int J Gynecol Cancer 29: 195-200, 2019.

12. Yang Z, Zhao B and Li L: The significance of the change pattern of serum CA125 level for judging prognosis and diagnosing recurrences of epithelial ovarian cancer. J Ovarian Res 9: 57, 2016.

13. Schummer M, Drescher C, Forrest R, Gough S, Thorpe J, Hellström I, Hellström KE and Urban N: Evaluation of ovarian cancer remission markers HE4, MMP7 and Mesothelin by comparison to the established marker CA125. Gynecol Oncol 125: 65-69, 2012.

14. Dorigo O and Berek JS: Personalizing CA125 levels for ovarian cancer screening. Cancer Prev Res (Phila) 4: 1356-1359, 2011.

15. Goel HL and Mercurio AM: VEGF targets the tumour cell. Nat Rev Cancer 13: 871-882, 2013.

16. Vempati P, Popel AS and MacGabhann F: Formation of VEGF isoform-specific spatial distributions governing angiogenesis: Computational analysis. BMC Syst Biol 5: 59, 2011.

17. Wada H, Ura S, Kitaoka S, Satoh-Asahara N, Horie T, Ono K, Takaya T, Takanabe-Mori R, Akao M, Abe M, et al: Distinct characteristics of circulating vascular endothelial growth factor-A and C levels in human subjects. PLoS One 6: e29351, 2011.
18. Wang $X$, Chen $X$, Fang $J$ and Yang C: Overexpression of both VEGF-A and VEGF-C in gastric cancer correlates with prognosis, and silencing of both is effective to inhibit cancer growth. Int J Clin Exp Pathol 6: 586-597, 2013.

19. Li L, Wang L, Zhang W, Tang B, Zhang J, Song H, Yao D, Tang Y, Chen X, Yang Z, et al: Correlation of serum VEGF levels with clinical stage, therapy efficacy, tumor metastasis and patient survival in ovarian cancer. Anticancer Res 24: 1973-1979, 2004

20. Lan Z, Fu D, Yu X and Xi M: Diagnostic values of osteopontin combined with CA125 for ovarian cancer: A meta-analysis. Fam Cancer 15: 221-230, 2016.

21. Ramchandani D and Weber GF: Interactions between osteopontin and vascular endothelial growth factor: Implications for cancer. Biochim Biophys Acta 1855: 202-222, 2015.

22. Wang YD, Chen H, Liu HQ and Hao M: Correlation between ovarian neoplasm and serum levels of osteopontin: A meta-analysis. Tumour Biol 35: 11799-11808, 2014.

23. Rao G, Wang H, Li B, Huang L, Xue D, Wang X, Jin H, Wang J, Zhu Y, Lu Y, et al: Reciprocal interactions between tumorassociated macrophages and CD44-positive cancer cells via osteopontin/CD44 promote tumorigenicity in colorectal cancer. Clin Cancer Res 19: 785-797, 2013.

24. Kim JH, Skates SJ, Uede T, Wong KK, Schorge JO, Feltmate CM, Berkowitz RS, Cramer DW and Mok SC: Osteopontin as a potential diagnostic biomarker for ovarian cancer. JAMA 287: 1671-1679, 2002.

25. Tilli TM, Franco VF, Robbs BK, Wanderley JL, da Silva FR, de Mello KD, Viola JP, Weber GF and Gimba ER: Osteopontin-c splicing isoform contributes to ovarian cancer progression. Mol Cancer Res 9: 280-293, 2011. 